\title{
FERRAMENTA COMPUTACIONAL PARA A CONSTRUÇÃO DE ANALISADORES VIRTUAIS UTILIZANDO SISTEMAS HÍBRIDOS NEURO-FUZZY
}

\author{
L. DOMINGUES ${ }^{1}$, S. V. SARAIVA ${ }^{1}$, F. O. CARVALHO ${ }^{1}$ e L.C.M. ALMEIDA ${ }^{1}$ \\ ${ }^{1}$ Universidade Federal de Alagoas, Departamento de Engenharia Química \\ E-mail para contato: leonardodomingues1994@gmail.com
}

\begin{abstract}
RESUMO - Neste trabalho foram elaboradas estratégias na forma de ferramentas computacionais para a construção de sensores virtuais baseado em sistema híbrido neuro-fuzzy, em MATLAB®, materializadas com interfaces amigáveis. A ferramenta aborda pré-tratamento estatístico de dados, normalização, preparação, treinamento e avaliação do sensor virtual. Uma validação foi realizada com dados referentes a uma coluna debutanizadora disponibilizados na literatura e esta se mostrou eficiente na inferência da concentração de butano na previsão de um tempo à frente, pois os dados simulados se ajustaram aos experimentais e os regressores explicaram praticamente toda a variável dependente.
\end{abstract}

\section{INTRODUÇÃO}

A inferência de variáveis, detecção e classificação de falhas, previsão de desempenho, aproximação de funções não lineares e identificação de padrões são problemas típicos da indústria e da investigação de fenômenos em geral. Em virtude da dificuldade operacional ou até de um alto custo dos sensores para monitoramento de uma variável desejada, surgem como uma boa alternativa em relação aos sensores tradicionais, os analisadores virtuais.

O analisador virtual consiste em um modelo utilizado para estimar variáveis de interesse de difícil medição em função de variáveis secundárias de fácil medição que podem estar presentes no controle e acompanhamento de processos químicos e ambientais. Na obtenção destes modelos são usados como dados de entrada os valores das variáveis que influenciam a variável desejada. A utilização de analisadores virtuais para estimar uma variável da planta para a qual nenhum sensor é instalado fornece uma oportunidade para melhorar o desempenho da mesma. O elemento mais importante deste, é o tipo de modelo da planta que permite a geração de uma medida estimada para substituir as medidas reais que são de difícil medição.

Existem muitas técnicas utilizadas para estimar variáveis através de outras variáveis medidas (inferência). De acordo com Ljung (1987) e também Ohshima e Tanigaki (2000), essas técnicas podem ser classificadas em dois grandes grupos: Modelos Fenomenológicos obtidos através de uma abordagem analítica, são os chamados modelos mecanicistas, derivados dos princípios básicos ou fundamentais do balanço de massa e energia; Modelos 
Caixa-Preta - obtidos de dados operacionais através da aplicação de algoritmos como redes neurais, sistemas híbridos neuro-fuzzy, computação nebulosa e/ou métodos estatísticos, tais como análise multivariável e PLS (Partial Least Squares).

Os modelos baseados em sistemas inteligentes como redes neurais e sistemas híbridos neuro-fuzzy vêm se destacando principalmente para processos e fenômenos altamente complexos como pode ser observado em Fortuna et al. (2007), o qual afirma que a construção de analisadores virtuais por estas técnicas apresenta uma vantagem no que diz respeito à construção do modelo fenomenológico visto que não é necessário nenhum conhecimento acerca do processo. A modelagem pode ser realizada através de um "aprendizado" assistido, onde através de aprendizagem, o sistema baseado em sistemas inteligentes faz a estimação das variáveis desejadas a partir de um histórico de dados de um domínio do processo.

Para estimar variáveis através de outras variáveis medidas, baseou-se no modelo de sistemas inteligentes híbrido neuro-fuzzy, pois este realiza a combinação da lógica fuzzy (LF) com as redes neurais (RNs) associando, assim, duas principais características inerentes a cada uma das técnicas: a capacidade de realizar raciocínio aproximado (LF) e a capacidade de aprendizagem (RNs). Desta forma, neste trabalho foram desenvolvidas estratégias na forma de ferramentas computacionais para a construção de um analisador virtual baseado em sistema híbrido neuro-fuzzy, abordando neste um pré-tratamento estatístico de dados até a normalização, preparação, criação, treinamento e avaliação do analisador virtual. A ferramenta computacional construída em ambiente MATLAB ${ }^{\circledR}$ é composta de interfaces amigáveis que permitem a utilização do sensor virtual de forma simples.

\section{METODOLOGIA}

A estratégia e o conjunto de ferramentas construídas (Figura 1) constitui um sistema computacional que engloba desde a melhor visualização e pré-tratamento dos dados passando pela detecção de outliers e por fim, a avaliação do treinamento como esquematizado na Figura 1.

Figura 1 - Sistema computacional construído.

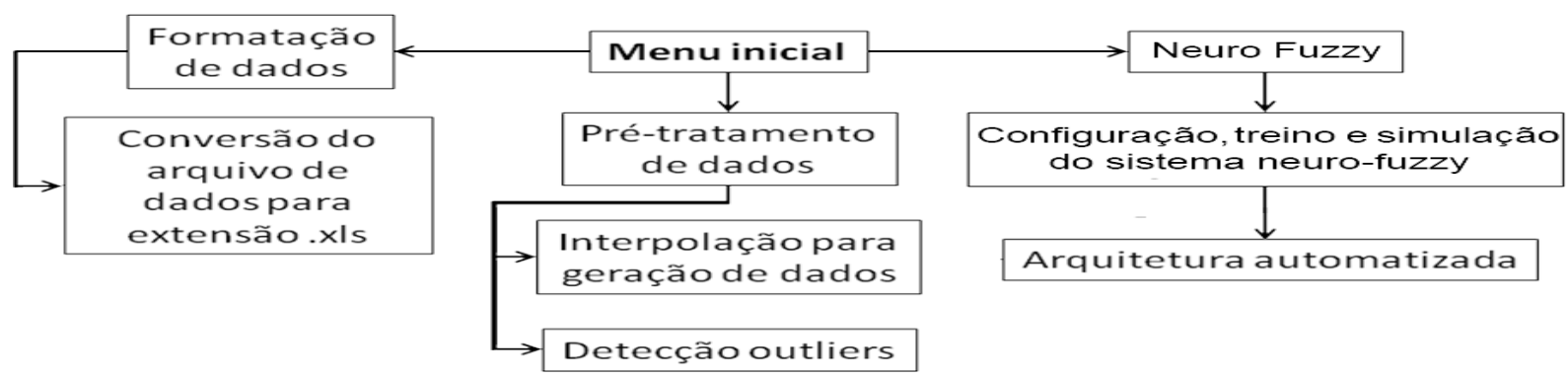

No pré-tratamento de dados especial atenção deve ser dada à coleta e análise dos dados visto que dados faltosos ou outliers podem prejudicar a eficiência do modelo desenvolvido.

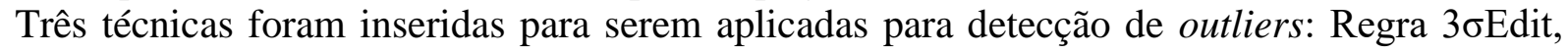
Parâmetros de Jolliffe e Análise Residual de Regressão Linear Múltipla.

Para melhor entendimento do sistema neuro-fuzzy, serão resumidas as técnicas 
utilizadas nesta ferramenta, a Lógica Fuzzy e as Redes Neurais. Podemos definir objetivamente a LF como sendo uma ferramenta capaz de capturar informações vagas, em geral descritas em uma linguagem natural e convertê-las para um formato numérico, de fácil manipulação pelos computadores.

A rede neural é um programa de computador que tenta imitar a intrincada estrutura de funcionamento do cérebro humano, refletindo no comportamento de uma máquina que aprende situações de causa-efeito. Redes neurais artificiais são compostas de vários elementos computacionais simples (nodos ou neurônio) que interagem localmente (altamante conectada). A arquitetura destes modelos é especificada pelas características do neurônio, topologia da rede e algoritmo de treinamento.

A modelagem utilizando os sistemas neuro-fuzzy é, atualmente, uma das técnicas mais difundidas na engenharia, principalmente quando aplicada a sistemas não lineares e de comportamento transiente, de acordo com Silva (2014). Segundo Oliveira Júnior et al.(2007), o sistema neuro-fuzzy representa o sistema fuzzy na forma de redes sujeitas a treinamento, por técnicas análogas às RNs. O método de treinamento é o ajuste de parâmetros, com a intenção de minimizar a função erro entre as saídas desejadas e as apresentadas pela rede.

Os três sistemas de sistemas inteligentes diferem entre si em alguns aspectos: sistema de inferência fuzzy aprende conhecimento dos dados somente através da LF; RN é capaz de aprender o conhecimento dos dados usando os pesos das conexões sinápticas; sistemas neurofuzzy são capazes de aprender o conhecimento de dados neurais com paradigma neural e representá-la na forma de regras nebulosas, de acordo com Mancini et.al. (2012).

Uma das redes neuro-fuzzy mais conhecidas e aplicadas é o sistema ANFIS (Adaptive Neuro-Fuzzy Inference System), introduzido por Jang (1993), e utilizada neste trabalho no ambiente MATLAB ${ }^{\circledR}$. Este sistema é um aproximador universal de função e, como tal, é capaz de aproximar qualquer função contínua real em um conjunto compacto para qualquer grau de precisão (Jang et al., 1997). O modelo ANFIS de sistema de suporte Takagi-Sugeno de primeira ordem admite múltiplas variáveis de entrada, porém, apenas uma variável de saída, dessa forma ele é identificado como um modelo de múltiplas entradas e única saída, onde os pesos entre as regras são iguais a uma unidade.

Para validação da ferramenta construída, foram utilizados dados de uma coluna debutanizadora obtidos da literatura de Fortuna et al (2007). Uma debutanizadora é uma coluna de fracionamento, onde o butano e outros componentes mais leves da gasolina são removidos.

\section{RESULTADOS}

A coluna debutanizadora (Figura 2) faz parte de uma planta de dessulfurização separação de nafta, onde os produtos removidos da corrente de nafta são o butano e o propano. 
Figura 2 - Flowsheet da coluna debutanizadora (Fortuna et al, 2007).

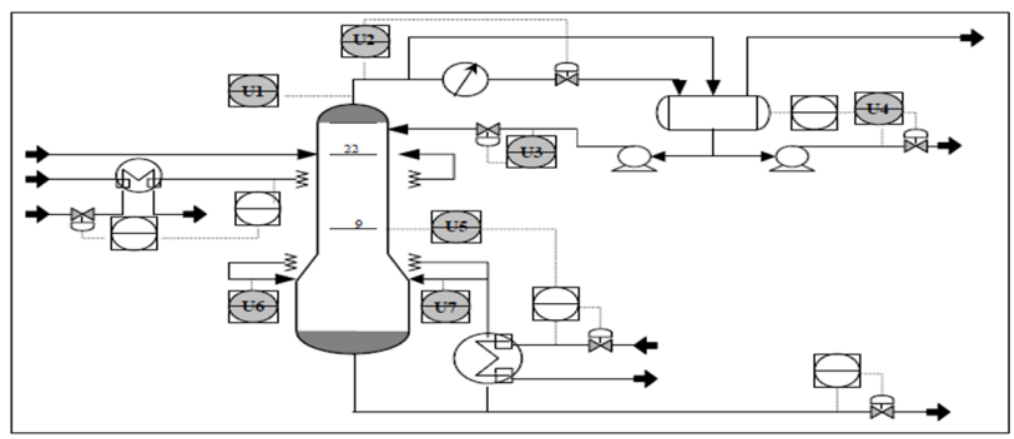

As variáveis usadas no treinamento são ilustradas na Figura 3 e indicam a utilização de um tempo de defasagem na previsão de um tempo à frente. Esta forma de utilização do analisador virtual está relacionada também com sua utilização em estratégias de controle avançadas de processos.

Figura 3 - Ilustração da rede utilizando o sistema ANFIS.
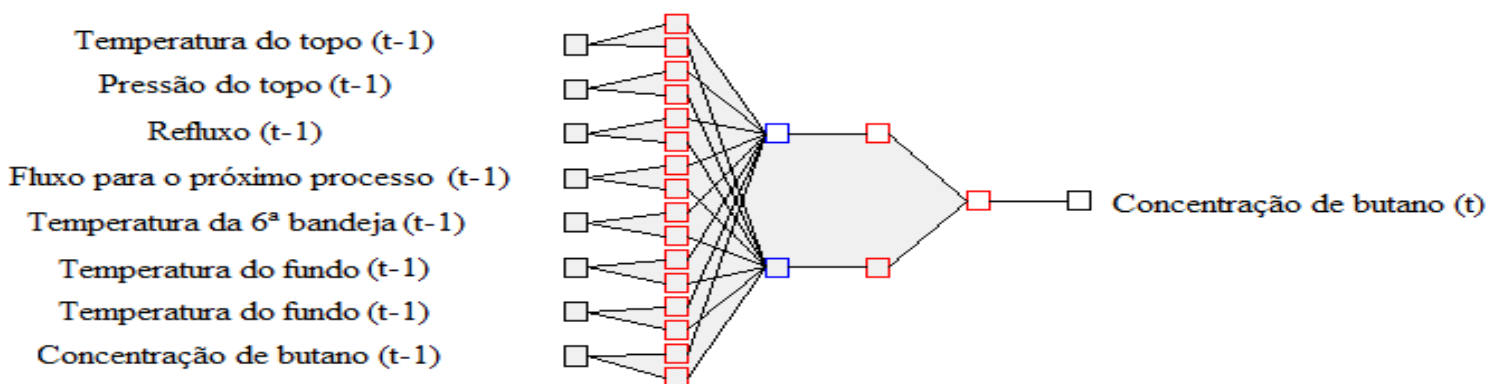

A primeira etapa de tratamento desses dados foi a busca e remoção de outliers. Todas as técnicas citadas anteriormente foram utilizadas simultaneamente para busca, resultando em um total de 8 outliers. O tratamento dado a estes foi a extração e interpolação.

Os dados foram testados na ferramenta construída através de interface amigável a qual está ilustrada na Figura 4, onde existe a possibilidade do ajuste de parâmetros para melhoria da eficiência da técnica. 
Figura 4 - Ilustração da interface com os parâmetros utilizados

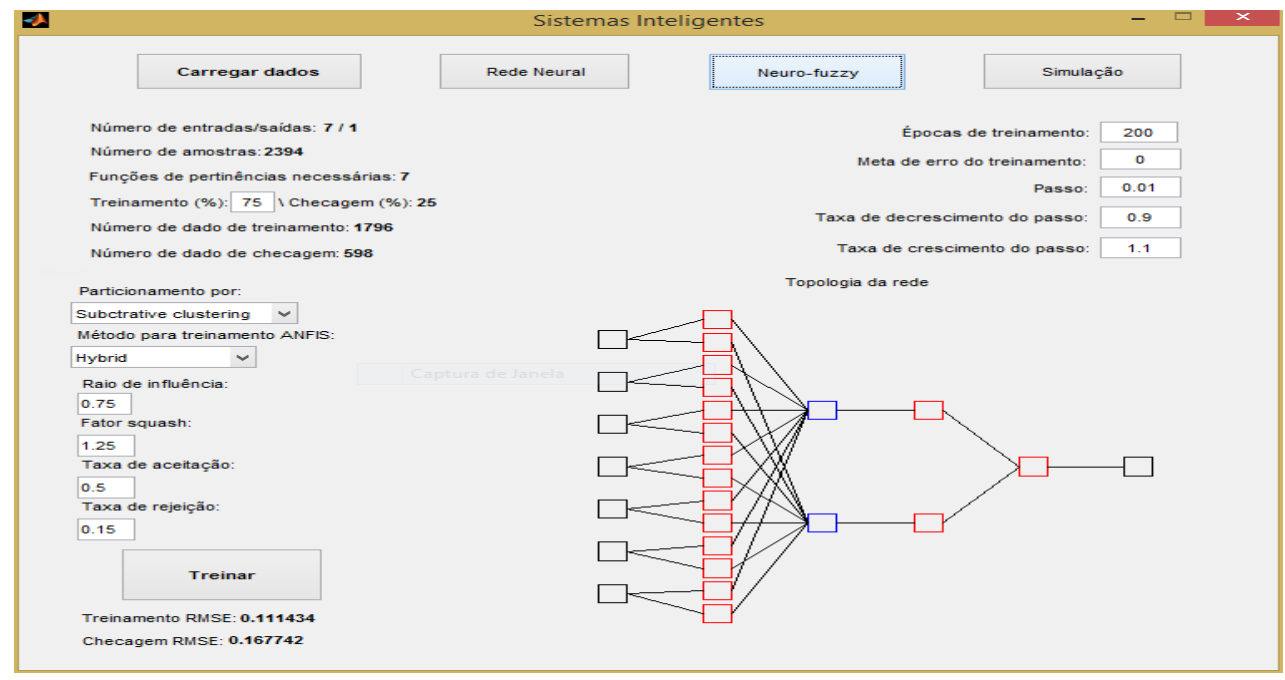

Os resultados do treinamento são apresentados na Figura 5.

Figura 5 - Resultado do treinamento do sistema ANFIS com os dados da debutanizadora. (a)

Gráfico do tipo amostras x valor da variável, onde a bola azul representa os dados experimentais e os asteriscos vermelhos os dados simulados. (b) Reta de regressão com coeficiente de 0.998 .

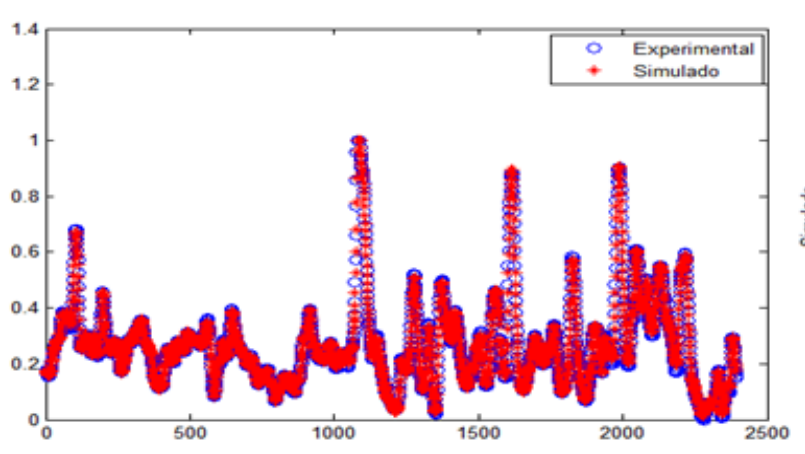

(a)

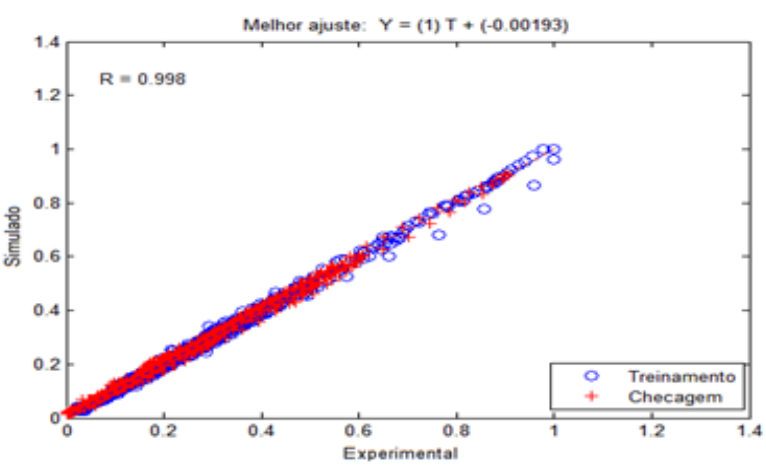

(b)

Observando a Fig. 5 (b), nota-se que houve um bom nível de representatividade do modelo visto que os regressores presentes no modelo explicam 99,8\% da variável dependente e que os dados simulados se ajustaram aos dados experimentais, segundo Fig. 5(a).

\section{CONCLUSÃO}

A ferramenta construída apresentou-se acessível e com bons resultados em relação aos dados de uma coluna debutanizadora. Esta se apresentou eficaz no desenvolvimento de analisadores virtuais para modelagem de processos e inferência de variáveis. Desta forma, conclui-se que a ferramenta pode ser aplicada a diferentes processos. 


\section{REFERÊNCIAS}

FORTUNA, L., GRAZIANI, S., RIZZO, A., XIBILIA, M. G. Soft Sensors for Monitoring and Control of Industrial Processes. Springer. 2007.

JANG, J.-S.R. ANFIS: adaptive-network-based fuzzy inference system. IEEE Transactions System Management and Cybernetics 23 (3), 665-685, 1993.

JANG, J.-S.R., SUN, C.-T. ET AL. Neuro-Fuzzy and Soft Computing: A Computational Approach to Learning and Machine Intelligence. Prentice Hall, Upper Saddle River, New Jersey, USA, 1997.

LJUNG, L. System Identification: Theory for the User. New Jersey: Prentice-Hall, 1987.

OHSHIMA, M.; TANIGAKI, M. Quality control of polymer production processes. Journal of Process Control, v.10, n.2-3, p.135-148, 2000.

MANCINI ,I. M., MASI, S., CANIANI, D., LIOI, D. S.. Fuzzy Logic and Neuro-Fuzzy Networks for Environmental. Hazard Assessment, Published in print edition March, 2012.

OLIVEIRA JUNIOR, H. A., CALDEIRA, A. M., et al., Inteligência Computacional aplicada à Administração, Economia e Engenharia em MATLAB. São Paulo: Thomson Learning, 2007.

SILVA, I. A. F. Aplicações de redes neurais e neuro fuzzy em engenharia biomédica e agronomia. Tese de Doutorado na Universidade Estadual Paulista, Faculdade de Engenharia, 2014. 